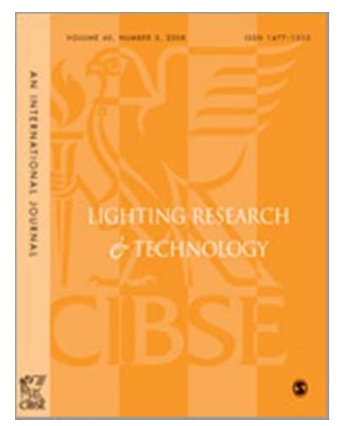

\title{
Physiological basis for visual discomfort: Application in lighting design
}

\begin{tabular}{|r|l|}
\hline Journal: & Lighting Research \& Technology \\
\hline Manuscript ID & LRT-15-0099.R1 \\
\hline Manuscript Type: & Review \\
\hline Date Submitted by the Author: & n/a \\
\hline Complete List of Authors: & Wilkins, Arnold; University of Essex, Psychology \\
\hline Keywords: & $\begin{array}{l}\text { visual stress, patterns, natural images, discomfort, flicker, saccadic } \\
\text { suppression, migraine }\end{array}$ \\
\hline Abstract: & $\begin{array}{l}\text { Visual discomfort occurs when the statistics of the retinal image depart } \\
\text { from those of natural scenes, particularly in respect of an excess energy at } \\
\text { spatial frequencies close to 3 cycles/degree. Computer models suggest that } \\
\text { uncomfortable stimuli are processed with a larger and less sparse neural } \\
\text { response. Uncomfortable stimuli usually evoke a relatively large } \\
\text { oxyenation of the visual cortex of the brain, consistent with inefficient } \\
\text { neural encoding. The discomfort may be homeostatic. The neural } \\
\text { computation that sustains sight is therefore likely to be more complex } \\
\text { when the visual scene is spatially periodic, when the colour contrast is high } \\
\text { or when saccadic suppression is impaired by flicker that is too rapid to be } \\
\text { seen. }\end{array}$ \\
\hline
\end{tabular}




\title{
Physiological basis for visual discomfort: Application in lighting design
}

\section{Keywords} \\ Address for correspondence \\ Department of Psychology \\ University of Essex \\ Colchester CO4 3SQ \\ 01206873333 \\ arnold@essex.ac.uk
}

Arnold J Wilkins DPhil

University of Essex, Colchester, UK

visual stress, patterns, natural images, discomfort, flicker, saccadic suppression, migraine 


\begin{abstract}
Visual discomfort occurs when the statistics of the retinal image depart from those from natural scenes, particularly in respect of an excess energy at spatial frequencies close to 3 cycles/degree. Computer models suggest that uncomfortable stimuli are processed with a larger and less sparse neural response. Uncomfortable stimuli usually evoke a relatively large oxygenation of the visual cortex of the brain, consistent with inefficient neural encoding. The discomfort may be homeostatic. The neural computation that sustains sight is therefore likely to be more complex when the visual scene is spatially periodic, when the colour contrast is high or when saccadic suppression is impaired by flicker that is too rapid to be seen.
\end{abstract}




\section{Introduction}

We will demonstrate that visual discomfort can be caused by images in which the spatial, chromatic and temporal features depart from those usually found in nature.

Natural scenes have a particular spatial structure -the complexity of the image remains the same across spatial scale. The Fourier spectrum decreases in amplitude with increasing spatial frequency. In many natural images, this decrease in amplitude is approximately proportional to the reciprocal of spatial frequency (1/f).

\section{INSERT FIGURE 1 ABOUT HERE}

A plot of amplitude against spatial frequency on log-log coordinates therefore has a slope close to $-1^{1-5}$, see Figure 1.

The chromatic structure of images of nature is constrained and colour contrasts are modest. ${ }^{6}$ The temporal variation in brightness is largely circadian. ${ }^{7}$ We will explore each of these natural attributes in turn.

\section{Spatial structure of images}

\subsection{Neural computation}

Given that the visual system has adapted to process natural images, one might expect that images with the spatial structure exemplified in Figure 1 would be computationally easy for the visual system to process. This expectation is borne out in several ways.

Visual processing is more efficient when images have a $1 / \mathrm{f}$ amplitude spectrum. ${ }^{2}$ The human contrast sensitivity function is optimised for encoding images with this structure. ${ }^{8}$ Also, the receptive fields of neurons in the primary visual cortex are such that images with $1 /$ f structure produce a sparse cortical response. ${ }^{9}$ The defining characteristic of this sparse response is that few neurons are active while many are inactive, thereby reducing metabolic demand. Hibbard and $\mathrm{O}^{\prime} \mathrm{Hare}^{10}$ have used a computational model of visual area V1 to show that uncomfortable stimuli such as striped patterns, which are rare in nature and do not conform to a $1 / f$ structure, result in an excess of "neural activity" and a non-sparse distribution of "neural" firing. Penacchio, Otazu, Wilkins, and Harris ${ }^{11}$ have extended this finding using a more elaborate model that includes the excitatory and inhibitory connections between neurons. They show that the sparseness of the distribution of neural firing correlates negatively with discomfort.

\subsection{Visual discomfort and $1 / \mathrm{f}$}

The above theoretical work therefore suggests that images are processed inefficiently by the brain if they do not posses a $1 / \mathrm{f}$ structure. Images of this kind are usually uncomfortable to 
look at. Juricevic, Land, Wilkins and Webster ${ }^{12}$ asked observers to rate the discomfort from meaningless images composed of filtered noise or randomly disposed randomly sized rectangles. For both categories of image, the discomfort was minimal with a $1 / f$ Fourier amplitude spectrum i.e. when the slope was -1 on log-log coordinates; the central pattern in Figure 2 has a slope of -1 . The discomfort increased when the slope was greater or less than -1 , as in the flanking patterns.

Fernandez and Wilkins ${ }^{13}$ showed that it is not simply the slope of the amplitude spectrum that is critical in determining discomfort. A variety of observers viewed images of nonrepresentational modern art. Again, images with a $1 / \mathrm{f}$ spectrum were rated as comfortable to look at. In this experiment, however, the uncomfortable images had a spectrum that departed from $1 / f$ in terms of the shape, not the slope, of the Fourier amplitude spectrum. The uncomfortable art had a curvilinear spectrum with an excess (relative to 1/f) of contrast energy at mid-range spatial frequencies. The human visual system is generally most sensitive to mid-range spatial frequencies, those within an octave of 3 cycles per degree. ${ }^{14}$ Using artificial images made by filtering random noise, Fernandez and Wilkins ${ }^{13}$ (2008) confirmed that departures from $1 / f$ were responsible for discomfort if the spatial frequency was close to 3 cycles per degree. By exchanging the phase and amplitude of comfortable and uncomfortable images they showed that the discomfort was determined by the amplitude rather than the phase information. O'Hare and Hibbard ${ }^{15}$ used images constructed from filtered noise and controlled for the apparent contrast of the stimuli. They also found that an excess of energy at mid spatial frequencies determined discomfort ratings, although with a spatial frequency tuning that was slightly lower than that obtained by Fernandez and Wilkins. ${ }^{13}$

\section{INSERT FIGURE 2 ABOUT HERE}

The Fourier amplitude spectrum is two-dimensional - it reflects the periodicity of the images at all orientations (vertical, horizontal and all orientations in between). The studies described above measured the Fourier amplitude spectrum by averaging over all orientations, as in Figure 1. Averaging over orientations loses the distinction between periodicity in one orientation and that in another. Wilkins et al. ${ }^{16}$ showed that checkerboards (which have contrast energy in several orientations) are less uncomfortable than stripes in which the energy varies only in one orientation. Penacchio and Wilkins ${ }^{17}$ therefore measured the Fourier amplitude in two dimensions. Instead of averaging over all orientations and fitting a straight line on log-log coordinates, as had previously been done, they fitted a cone with slope of -1 to the two-dimensional log amplitude spectrum. The residual error in the fit provided a useful index that could reliably predict how uncomfortable the image was. The residual error increased as the structure of the image departed from that expected for a natural image. Penacchio and Wilkins ${ }^{17}$ used seven sets of images: photographs of everyday scenes, of buildings, of animals, images of randomly generated polka dots and non-representational art. All the images were rated for discomfort. Despite the large range of images, the index explained $17 \%$ of the variance in judgments of discomfort. The prediction was improved when 


\section{Lighting Research \& Technology}

A J Wilkins PHYSIOLOGICAL BASIS FOR VISUAL DISCOMFORT

the residuals were weighted to take account of the greater sensitivity to mid-range spatial frequencies, as reflected in a published estimate of the contrast sensitivity function. ${ }^{18}$ From these two principles gleaned from the literature (without fitting any parameters) they were able to explain an average of $27 \%$ of the variance in judgments of discomfort of a wide range of images. This is surprising given the variation between people. Figure 3 shows an example of an image that is recognized by the algorithm as uncomfortable, and one that is comfortable.

In summary, two related factors were found to predict judgments of discomfort: 1. departure from the statistics of natural images, and 2. excess energy at the spatial frequencies to which the human visual system is generally most sensitive, i.e about 3 cycles per degree.

Using grating patterns with this spatial frequency Wilkins et $a l^{16}$ showed that the discomfort increased linearly with the spatial extent of the visual cortex to which the pattern projected. The discomfort is therefore determined not only by the spatial frequency but by the size of a pattern.

\section{INSERT FIGURE 3 ABOUT HERE}

\section{Hypermetabolism and discomfort}

We have seen that images with Fourier amplitude spectra that depart from $1 / f$ are not efficiently processed and are uncomfortable to look at. In theory, at least, they involve a less sparse coding and a greater neural response overall. As will now be shown, the theory is supported by physiological evidence that the neural response to these uncomfortable images is indeed greater than to images that are comfortable.

When a visual stimulus is observed, there is a change in the oxygenation of the blood reaching the visual cortex - the cortical haemodynamic response. The cortical haemodynamic response to visual stimuli reflects the activity of large numbers of neurons and their local collective demand for oxygenated blood. The relationship between the amplitude of the haemodynamic response and the size of the neuronal response is complex and indirect. It is affected by many factors such as blood flow and glial cell activity, but generally broadly reflects local field potentials. ${ }^{19}$ The response can be measured using functional magnetic resonance imaging (fMRI) and near infrared spectroscopy (NIRS). As we will now see, both techniques show that the oxygenation is greater when the visual stimulus is uncomfortable.

Haigh et al. ${ }^{20}$ used NIRS of the visual cortex and found that coloured patterns gave a larger oxyhaemoglobin response if they had large differences in their component colours and were therefore uncomfortable to view. Huang et al. ${ }^{21}$, measured the Blood Oxygen Level Dependent (BOLD) response to achromatic gratings with a range of spatial frequencies and showed that those with mid spatial frequency (which are uncomfortable) gave the largest response.

It is also quite generally the case that individuals who are susceptible to discomfort show a larger BOLD response than those who are not. Huang et al. ${ }^{21}$ showed that patients with 
migraine who reported high levels of discomfort from patterns gave a BOLD response with relatively high amplitude. Martin et al. ${ }^{22}$ compared 19 patients with migraine and 19 controls. Patients with migraine had a larger number of activated occipital voxels than controls. Cucchiara et al. ${ }^{23}$ found that in migraine patients who experienced aura the number of symptoms of discomfort they reported by questionnaire correlated with the amplitude of the BOLD response to visual stimulation.

Although the studies reviewed in the above paragraph concerned patients with migraine, the relationship between discomfort and the size of the haemodynamic response occurs independently of this diagnosis. Thus, Alvarez-Linera Prado et al. ${ }^{24}$ compared 20 photophobic patients with 20 controls who viewed a light source at various intensities. There was a direct relationship between stimulus intensity and the size of the BOLD response, and the response was higher in the photophobic patients, particularly at low and medium light intensities. Chouinard, Zhou, Hrybouski, Kim, and Cummine ${ }^{25}$ reported a case study of an individual with visual stress. The BOLD response was measured when lists of words were read, and an elevated activity was found in a variety of visual and somatosensory areas. Bargary, Furlan, Raynham, Barbur, and Smith ${ }^{26}$ compared normal participants with high and low discomfort glare thresholds while they identified the orientation of a Landolt $C$ surrounded by peripheral sources of glare. The group that was sensitive to discomfort glare had an increased BOLD response localized at three discrete bilateral cortical locations: in the cunei, the lingual gyri and in the superior parietal lobules.

There is therefore a relationship between discomfort and the magnitude of the haemodynamic response in the visual cortex both in terms of the stimuli that evoke discomfort, which generally induce a large response, and in terms of the individuals who are susceptible to discomfort, in whom the response is larger than in others. It is possible that the discomfort is homeostatic. As with any other pain, it encourages withdrawal and thereby acts to reduce the use of energy by the brain. The brain constitutes $2 \%$ of body weight but consumes $20 \%$ of the body's energy. Only a small fraction, perhaps $1 \%$, of the cerebral cortex can be supplied with energy and be active at any given time,$^{27,28}$ so conservation of metabolic energy is an important requirement.

\section{Colour contrasts and light source chromaticity}

So far, consideration has been limited to patterns of luminance but we now consider differences in colour. Haigh et al. ${ }^{20}$ measured the discomfort from gratings with bars that alternated between two colours. They showed that discomfort from these patterns was predicted from the separation of the chromaticities in the CIE 1976 UCS diagram: the larger the separation, the greater the discomfort and the larger the haemodynamic response evoked. This was the case in many studies and for a large gamut of colours, some with different luminance. Juricevic et al. ${ }^{12}$ also showed that discomfort was greater for images with a large colour difference. They used images comprising random dots or randomly disposed 
rectangles and measured the colour difference in terms of the $L-M$ and $S-L M$ chromatic plane. Large colour contrasts are rare in the natural world, ${ }^{6,12}$ so once again, in both the studies by Haigh et al. ${ }^{20}$ and Juricevic et al., ${ }^{12}$ discomfort was associated with images that are rare in nature. Large colour contrasts are not necessarily rare in the modern urban environment, as Figure 4 shows. It is not yet known whether large colour contrasts in day-today settings can give rise to discomfort, but a comparison of the left and right images in Figure 4 suggests that they might.

Such colour contrasts are, of course, affected by the spectral power distribution of the lighting, and this in itself can be responsible for discomfort. There are large individual differences in people's preference for the colour of lighting, and these individual differences have a neurological basis. Huang et al. ${ }^{29}$ asked patients with migraine to observe text in an apparatus that permitted the separate control of the hue, saturation and luminance and the illuminating light. They selected a chromaticity that optimised the visual comfort of the page. Wearing coloured filters, they later observed patterns of stripes. Three filters were compared; one provided the chosen chromaticity, one provided a chromaticity that differed by about 6 jnd's and one simply reduced the luminance by an amount equivalent to the reduction afforded by the other two filters. When a stressful ( 3 cycles/degree) pattern was observed, the BOLD response was selectively reduced with the filter that provided the chosen chromaticity. The other filters had no such effect. The BOLD response to the filters did not differ when a non-stressful pattern (spatial frequency 0.5 cycles/degree) was observed. In migraine patients the BOLD response is usually abnormally large when stressful patterns are observed, ${ }^{21,29}$ so the reduction in the haemodynamic response with a coloured filter suggests a possible therapy for migraine, and helps to explain the strong aversion to colour schemes that people sometimes express.

\section{INSERT FIGURE 4 ABOUT HERE}

\section{Temporal characteristics}

Variation in brightness over time, when it is rapid, is usually called flicker. Flicker is perceptible at low frequencies. It is not only uncomfortable, but can cause seizures ${ }^{16}$. As frequency is increased there comes a point at which a flickering light appears steady, the socalled critical flicker fusion threshold (CFF). Even though the light may appear steady when its frequency is above the CFF, the flicker may nevertheless have perceptible effects on the appearance of a moving target (stroboscopic effect), and movements of the eyes may give rise to a perceptible array of multiple images (phantom array). These perceptible effects are spatial and are the combined effect of the temporal variation in brightness and target displacement on the retina. Because the eyes move at up to 700 degrees per second during a saccade (rapid jerk of the eye) the spatial effects of flicker can be perceived at frequencies in the kilohertz range. ${ }^{30}$ 
When our eyes make a saccade we are usually unaware of the motion of the image across the retina. This is partly because of active neural mechanisms of suppression, ${ }^{31}$ partly because of masking by the images before and after the saccade, ${ }^{32}$ but also partly because the motion of the image on the retina is continuous and its velocity is outside the range that occurs during fixation, to which the neurons are most sensitive. ${ }^{33}$ Under flickering illumination the image on the retina is not continuous but a series of discrete images; the normal mechanisms of saccadic suppression then break down, and the intrasaccadic images can sometimes be perceived. This is particularly noticeable at night when automobile backlights use LEDs that are lit periodically. A trail of lights is visible with each saccade. This is a distraction: the normal processes of computation that sustain perception are rendered more complex. Such interference with saccadic suppression may be one reason why supra-CFF flicker has been shown to interfere with eye movements ${ }^{34,35}$ and to induce headaches. Wilkins et al. $^{36}$ studied the daily incidence of headaches and eye-strain in office workers over a fivemonth period. Halfway through this period the circuitry controlling the fluorescent lighting in the offices was changed from low frequency wire-wound ballast to high frequency solid-state ballast and vice versa. The change occurred without the awareness of the office occupants, half of whom received the low frequency lighting first, and half second. The low frequency ballast gave light that varied at $100 \mathrm{~Hz}$ by about $35 \%$ of maximum. The high frequency ballast had little variation at $100 \mathrm{~Hz}$. The incidence of headaches was halved under the high frequency ballast. The reduction was due largely to the few occupants who suffered frequent headaches.

\section{INSERT FIGURE 5 ABOUT HERE}

\section{Application to lighting design}

\subsection{Spatial configuration}

We have shown that visual images from nature are processed efficiently and comfortably. When the spatial characteristics of an image are unnatural the neural processing is inefficient, metabolic demand increases and discomfort ensues. The discomfort increases with the spatial extent of the visual cortex to which the stimulus projects, larger patterns being more uncomfortable. A simple algorithm described by Penacchio and Wilkins ${ }^{17}$ predicts discomfort from images on the basis of departures from $1 / f$ structure, particularly those that involve excess contrast energy at mid spatial frequencies. The algorithm should be helpful in avoiding discomfort from lighting design, as, for example, in the spatial arrangement of luminaires Figure 5 (left). Striped patterns such as shown here have Fourier amplitude spectra that depart maximally from $1 /$ f. Similar considerations apply to arrays of point sources on ceilings, and LED lamps on cars, Figure 5 (right), although the latter are small in area and therefore less uncomfortable. The discomfort depends both on the spatial frequency of the array and the retinal subtense (extent of the visual cortex to which the pattern projects) according to functions published by Wilkins et al. ${ }^{16}$ 


\subsection{Colour}

Both the light source chromaticity and colour contrasts within a living space affect comfort, but they do so individually, with large variations between people. Light sources and large surface areas with strongly saturated colour (particularly red) can induce headache. ${ }^{37}$ When lighting a space with a strong colour pattern that cannot be avoided, the effects of the pattern can be reduced by using a light source with a limited gamut, thereby reducing the colour saturation.

\subsection{Flicker}

Flicker in the range $4-60 \mathrm{~Hz}$ can induce seizures in a small proportion of the population with photosensitive epilepsy. It can also induce headaches. Seizures and headaches are most likely when the flicker is between 15 and $20 \mathrm{~Hz}^{38}$. Flicker in this frequency range is sometimes produced when $50 \mathrm{~Hz}$ compact fluorescent lamps ignite (author's measurements). If these lamps are used in public places with occupancy sensors they ignite automatically without warning and constitute an unacceptable hazard.

Flicker from fluorescent lighting is rarely visible as such. Nevertheless observers with high CFF are more likely to complain of fluorescent lighting ${ }^{39}$. Flicker can be appreciated at frequencies in the kilohertz range, well above the CFF. This is due to the patterns formed during rapid eye movements.

Although fluorescent lighting with the more efficient high frequency electronic ballast has now largely replaced the low frequency circuitry, the legacy of inefficient unhealthy lighting is still with us. In a survey of schools in Britain in $2009,80 \%$ of classrooms were found to be lit with low-frequency circuitry. ${ }^{40}$

\section{INSERT FIGURE 6 ABOUT HERE}

The contemporary challenge is to prevent the flicker from LED lighting - both flicker that is below the CFF and flicker that is above the CFF and therefore usually imperceptible but not necessarily innocuous. A recent survey of LED lamps available on the market has found that many flicker, and for some the variation is greater than for fluorescent lamps. ${ }^{41}$ Under these circumstances it seems likely that the introduction of LED lighting will be met with complaints and resistance similar to those that accompanied the introduction of compact fluorescent lighting (which also often flickered). IEEE has published guidance on the acceptable limits for flicker broadly similar to those in Figure 6 right. It is to be hoped that this guidance will help prevent the various negative health impacts that result from supra-CFF flicker, which have been summarized by Wilkins, Veitch and Lehman. ${ }^{43}$ Note that Paplowski and Miller ${ }^{41}$ express the variation in terms of the flicker index, which takes some account of the atypical waveforms from LED lamps, whereas Lehman and Wilkins ${ }^{42}$ express the variation in terms of the simpler percent modulation. It is possible that in due course both expressions can be subsumed in a formulation that applies appropriate weights to the Fourier series derived from the waveform by which the light varies over time. In unpublished work Drury and Wilkins have found that the 
visibility of intrasaccadic flicker is greater at $300 \mathrm{~Hz}$ than at $100 \mathrm{~Hz}$, presumably because the pattern formed on the retina during the flight of the eye has spatial characteristics that are closer to the peak of the spatial contrast sensitivity function ${ }^{14}$. This finding suggests that the recommendations of IEEE $1789^{42}$ are not conservative.

Paplowski and Miller ${ }^{41}$ summarised the conditions that contribute to a higher risk of adverse responses to flicker as follows: long exposure, large area of retina receiving stimulation, and high luminance. In all these respects exposure to lighting is of high risk, and the exposure cannot be avoided.

\section{Conclusion}

The human visual system evolved to process images from nature. In the modern urban environment the visual images it is required to process make the neural computation involved in sight more complex than it needs to be, with consequences for discomfort, cortical metabolism and, more generally, for health. The beneficial effects of exercise in natural surroundings ("green exercise" ${ }^{44}$ ) may perhaps be explained in these terms, as perhaps can other examples that support the so-called "biophylia hypothesis". ${ }^{45}$

The spatial configuration of lamps and luminaires is often such as to induce discomfort. The use of large areas of highly saturated colours can also cause discomfort for some individuals. It is a mistake to assume that a lamp is "flicker free" simply because the flicker cannot be seen. It is essential that the lighting industry does not repeat the mistakes of the past. Imperceptible flicker from lighting has adversely affected the lives of many, if not most individuals who suffer migraine ${ }^{45}$ and future generations should not have to bear this burden.

\section{Acknowledgements}

I thank Dr Peter Boyce for perspicacious comments on an earlier draft. 
A J Wilkins PHYSIOLOGICAL BASIS FOR VISUAL DISCOMFORT

\section{References}

1. Burton GJ, Moorhead IR. Color and spatial structure in natural scenes Appl. Opt. 1987; $26,157-70$

2. Field DJ. Relations between the statistics of natural images and the response properties of cortical cells. Opt. Soc. Am. A, 1987; 4(12), 2379-2394.

3. Field DJ. What is the goal of sensory coding? Neural Comput. 1994; 6 559-601.

4. Parraga CA, Brelstaff G, Troscianko T, Moorhead IR. Color and luminance information in natural scenes. Journal of the Optical Society of America A 1998; 15(3), 563-569.

5. Tolhurst DJ, Tadmore $\mathrm{Y}$, Tang $\mathrm{C}$. The amplitude spectra of natural images Ophthalmic Physiol. Opt. 1992; 12 229-232.

6. Webster MA, Mollon JD. Adaptation and the color statistics of natural images. Vision Res.1997; 37(23), 3283-3298.

7. Paul KN, Saafir TB, Tosini G, The role of retinal photoreceptors in the regulation of circadian rhythms. Rev Endocr Metab Disord, 2009; 10(4), 271-278.

8. Atick JJ Redlich AN. What does the retina know about natural scenes? Neural Computation 1992; 4, 196-210.

9. Olshousen B Field DJ. Sparse coding with an overcomplete basis set: a strategy employed by V1? Vision Research 1997; 37, 3311-3325.

10. Hibbard P O'Hare L. Uncomfortable images produce non-sparse responses in a model of primary visual cortex, Royal Society Open Science 2015; 2: 140535

11. Penacchio $O$, Otazu $X$, Wilkins AJ Harris J. Uncomfortable images prevent lateral interactions in the cortex from providing a sparse code. ECVP 2015 Perception in press.

12. Juricevic I, Land L, Wilkins AJ, Webster M. Visual discomfort and natural image statistics. Perception, 2010; 39(7), 884-899.

13. Fernandez D Wilkins AJ. Uncomfortable images in art and nature. Perception, 2008; 37(7), 1098-1113.

14. Campbell FW Robson JG. Application of Fourier analysis to the visibility of gratings. Journal of Physiology, 1968; 197, 551-566.

15. O'Hare L Hibbard PB. Spatial frequency and visual discomfort. Vision Research, 2011; 51, 1767-1777.

16. Wilkins AJ, Nimmo-Smith MI, Tait A, McManus C, Della Sala S, Tilley A et al. A neurological basis for visual discomfort. Brain,1984; 107, 989-1017.

17. Penacchio O Wilkins AJ. Visual discomfort and the spatial distribution of Fourier energy. Vis. Res. 2015; (in press).

18. Mannos JL Sakrison DJ. Effects of a visual fidelity criterion on encoding of images. IEEE Transactions on Information Theory., 1974; 20(4), 525-536.

19. Logothetis NK, Pauls J, Augath M, Trinath T, Oeltermann A. Neurophysiological investigation of the basis of the fmri signal. Nature, 2001; 412(6843), 150-157.

20. Haigh S, Barnigham L, Berntsen M, Coutts L, Hobbs E, Irabor et al. Discomfort and the cortical haemodynamic response to coloured gratings. Vision Research,2013; 30(89), 4753.

21. Huang J Cooper TG, Sarana D, Kaufman DI, Cao Y. Visual distortion provoked by a stimulus in migraine associated with hyperneuronal activity. Headache,2003; 43(6), 664671.

22. Martín H, Sánchez del Río M, de Silanes CL, Álvarez-Linera J, Hernández JA, Pareja JA. Photoreactivity of the occipital cortex measured by functional magnetic resonance imaging-blood oxygenation level dependent in migraine patients and healthy volunteers: pathophysiological implications. Headache, 2011; 51(10), 1520-1528.

23. Cucchiara B, Datta R, Aguirre GK, Idoko KE, Detre J. Measurement of visual sensitivity in migraine: Validation of two scales and correlation with visual cortex activation. . Cephalalgia. 2015; 35(7):585-92.

24. Alvarez-Linera Prado J, Ríos-Lago $M$, Martín-Alvarez $H$, Hernández-Tamames JA, Escribano-Vera J, Sánchez del Río M. Functional magnetic resonance imaging of the 


\section{Lighting Research \& Technology}

A J Wilkins PHYSIOLOGICAL BASIS FOR VISUAL DISCOMFORT

visual cortex: relation between stimulus intensity and bold response Revista de Neurologia 2007; 45(3):147-151.

25. Chouinard B, Zhou C, Hybrouski S, Kim E, Cummine J. A functional neuroimaging case study of Meares-Irlen syndrome/visual stress (misvis). . Brain Topogr. 2011; 25(3), 293307.

26. Bargary G, Furlan M, Raynham PJ, Barbur JL, Smith AT. Cortical hyperexcitability and sensitivity to discomfort glare. Neuropsychologia,2015; 69, 194-200.

27. Attwell D Laughlin SB. An Energy Budget for Signaling in the Grey Matter of the Brain. J Cereb Blood Flow Metab, 2001; 21(10), 1133-1145.

28. Howarth C, Gleeson P, Attwell D. Updated energy budgets for neural computation in the neocortex and cerebellum. Journal of Cerebral Blood Flow and Metabolism, 2012; 32, 1222-1232.

29. Huan J, Zong X, Wilkins A, Jenkins B, Bozoki A, Cao Y. fMRI evidence that precision ophthalmic tints reduce cortical hyperactivation in migraine.Cephalalgia $2011 ; 31(8), 925-$ 36.

30. Roberts JE, Wilkins AJ. Flicker can be perceived during saccades at frequencies in excess of $1 \mathrm{khz}$. Lighting Research and Technology, 2013; 45, 123-132.

31. Bremmer F, Kubischik M, Hoffmann KP, Krekelberg B. Neural dynamics of saccadic suppression. Journal of Neuroscience 2009; 29(40) 12374-12383.

32. Campbell FW, Wurtz RH. Saccadic omission: why we do not see a grey-out during a saccadic eye movement. Vision Research 1978; 18, 1297-1303.

33. Castet E, Masson GS. Motion perception during saccadic eye movements. Nature Neuroscience 2009; 3(2), 177-183.

34. Wilkins AJ. Intermittent illumination from visual display units and fluorescent lighting affects movements of the eyes across text. Human Factors, 1986; 28(1), 75-81.

35. Kennedy A, Murray WS. The effects of flicker on eye movement control. Q. J. Exp. Psychol, 1991; 43(1), 79-99.

36. Wilkins AJ, Nimmo-Smith I, Slater A, Bedocs L. Fluorescent lighting, headaches and eyestrain. Lighting Research and Technology, 1989; 21(1), 11-18.

37. Barbur JL Ruddock KH, Waterfield VA. A colour-dependent abnormality in human visual detection of stimulus motion and spatial structure. Neurosci.Lett. 1979; 15:307-312.

38. Wilkins AJ, Binnie CD, Darby CE. Visually-induced seizures. Progress in Neurobiology $1980 ; 15,85-117$.

39. Brundrett GW. Human sensitivity to flicker Lighting Research and Technology 1974; 6 127-143.

40. Winterbottom M, Wilkins AJ. Lighting and discomfort in the classroom. Journal of Environmental Psychology, 2009; 29, 63-75.

41. Paplowski ME, Miller NE. Flicker in solid-state lighting: measurement techniques, and proposed reporting and application criteria. Conference Proceedings of the CIE Midterm and Centenary Conference Paris 2013.

42. Lehman B, Wilkins AJ. Designing to mitigate the effects of flicker in LED lighting. IEEE Power Electronics Magazine, 2014; 1(3), 18-26.

43. Wilkins AJ, Weitch $J$ Lehman B. LED lighting flicker and potential health concerns: IEEE standard PAR1789 update. IEEE ECCE, 2010;171-178.

44. Pretty J, Peacock J, Sellens M, Griffin M. The mental and physical health outcomes of green exercise. International Journal of Environmental Health Research 2005;15 (5): 319-37.

45. Harle DE, Shepherd AJ Evans BJ. Visual stimuli are common triggers of migraine and are associated with pattern glare. Headache, 2006; 46(9), 1431-1440. 


\section{Lighting Research \& Technology}

A J Wilkins PHYSIOLOGICAL BASIS FOR VISUAL DISCOMFORT

\section{Figure Legends}

Figure $1-1 / f$ amplitude spectra for luminance and chrominance for the 29 scenes measured by Parraga et al. ${ }^{3}$

Figure 2 - Examples of meaningless patterns of filtered random dots. The slopes of the amplitude spectra (left-right) are $-2,-1.5,-1,-0.5$ and 0 (From Jurecevic et al. ${ }^{12}$ ).

Figure 3 - Examples of artwork by Debbie Ayles that is recognized as uncomfortable (left) and comfortable (right) by the algorithm of Penacchio and Wilkins. ${ }^{17}$

Figure 4 - (left) Strong colour contrasts in a primary school (Courtesy of EME Furniture, reproduced with permission); (right) same figure with contrasts reduced

Figure 5 - Uncomfortable configurations of luminaires and lamps

Figure 6 - Acceptable and unacceptable limits of flicker: left: from Paplowski and Miller (2013),; right: from Lehman and Wilkins (2014). Note the linear axes on the left figure and logarithmic axes on the right figure. Reproduced with permission. 
A J Wilkins PHYSIOLOGICAL BASIS FOR VISUAL DISCOMFORT

1

2

3

4

5

6

7

8

9

10

11

12

13

14

15

16

17

18

19

20

21

22

23

24

25

26

27

28

29

30

31

32

33

34

35

36

37

38

39

40

41

42

43

44

45

46

47

48

49

50

51

52

53

54

55

56

57

58

59

60

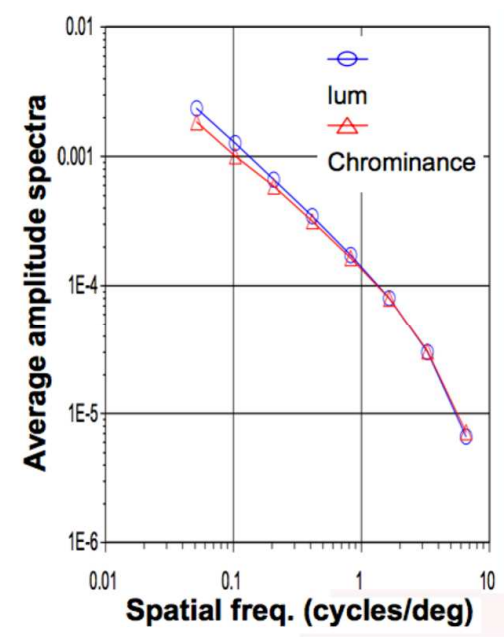

Figure 1 - 1/f amplitude spectra for luminance and chrominance for the 29 scenes measured by Parraga et al. ${ }^{3}$ 

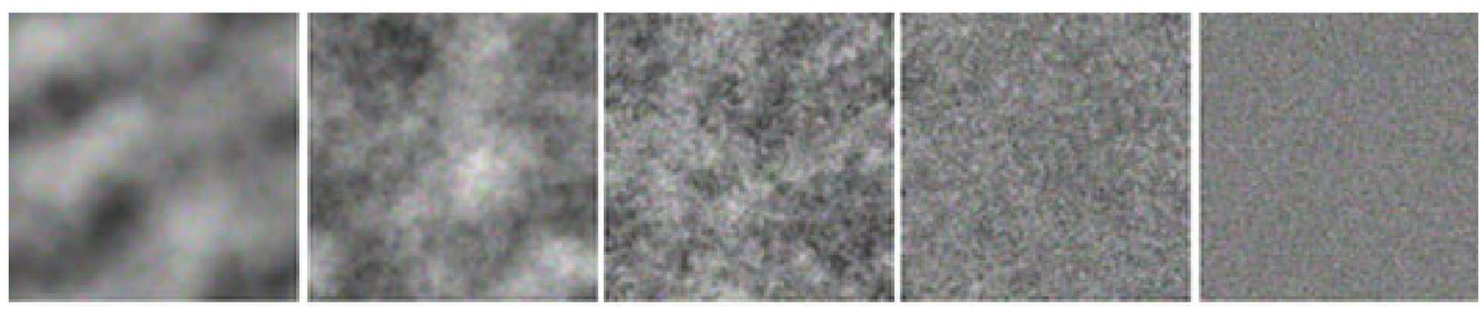

Figure 2 - Examples of meaningless patterns of filtered random dots. The slopes of the amplitude spectra (left-right) are $-2,-1.5,-1,-0.5$ and 0 (from Jurecevic et al. ${ }^{12}$ ). 


\section{Lighting Research \& Technology}

A J Wilkins PHYSIOLOGICAL BASIS FOR VISUAL DISCOMFORT
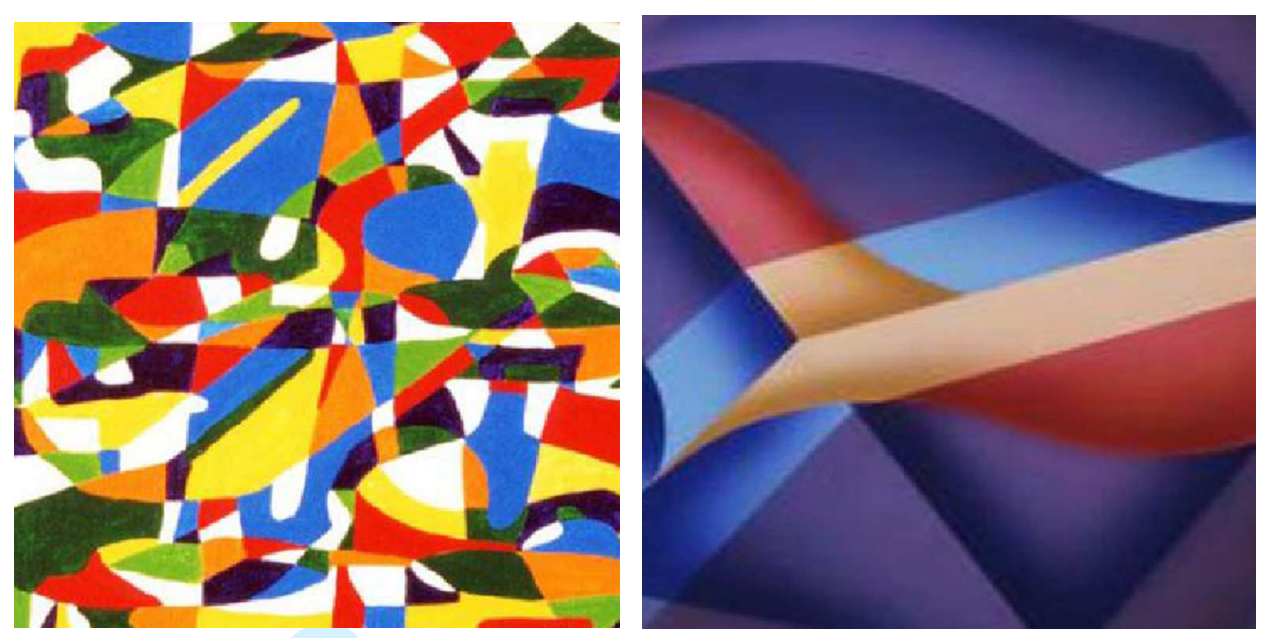

Figure 3 - Examples of artwork by Debbie Ayles that is recognized as uncomfortable (left) and comfortable (right) by the algorithm of Penacchio and Wilkins. ${ }^{17}$ 
A J Wilkins PHYSIOLOGICAL BASIS FOR VISUAL DISCOMFORT

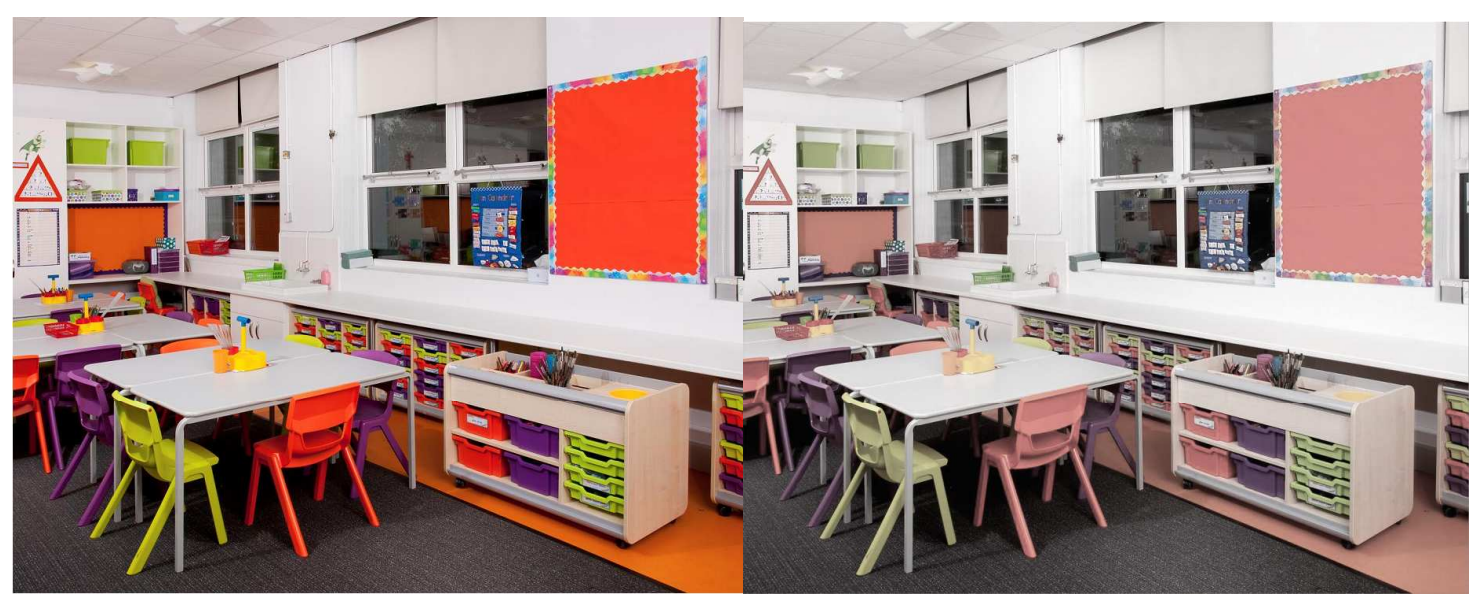

Figure 4 - (left) Strong colour contrasts in a primary school (Courtesy of EME Furniture, reproduced with permission); (right) same figure with contrasts reduced 
A J Wilkins PHYSIOLOGICAL BASIS FOR VISUAL DISCOMFORT
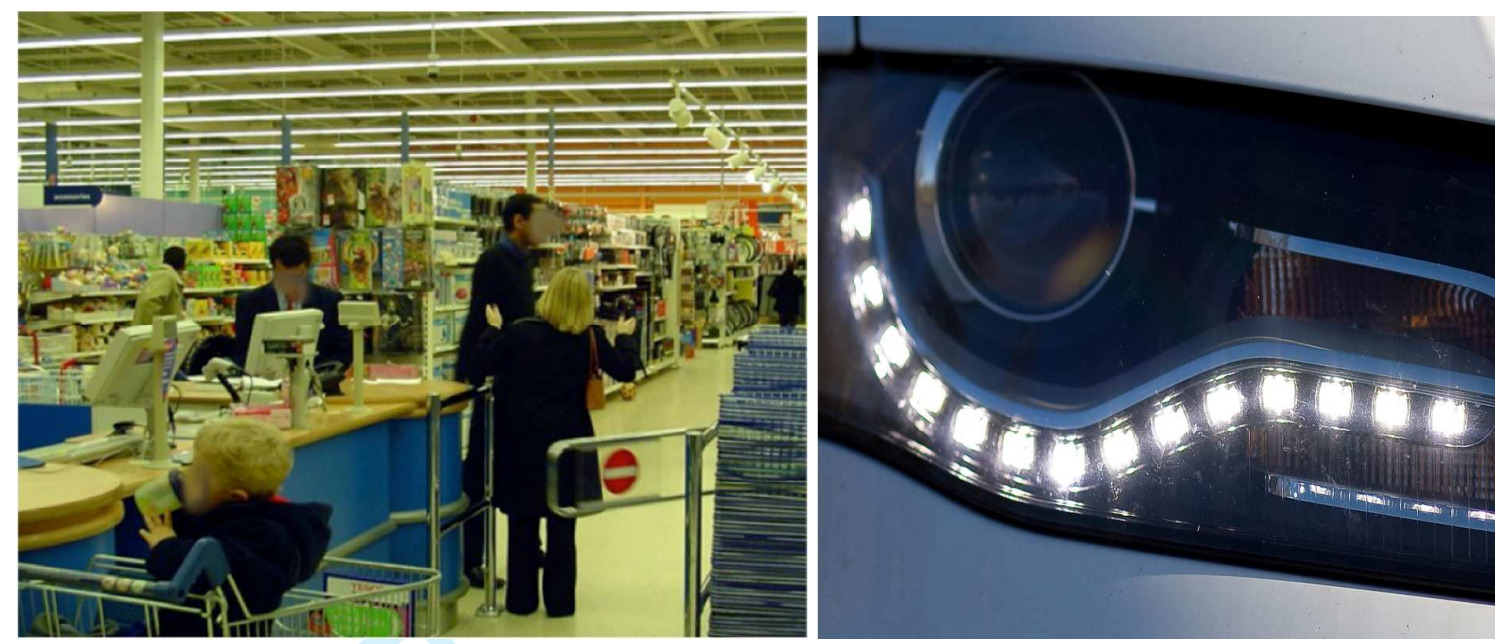

Figure 5 - Uncomfortable configurations of luminaires and lamps 
A J Wilkins PHYSIOLOGICAL BASIS FOR VISUAL DISCOMFORT

1

2

3

4

5

6

7

8

9

10

11

12

13

14

15

16

17

18

19

20

21

22

23

24

25

26

27

28

29

30

31

32

33

34

35

36

37

38

39

40

41

42

43

44

45

46

47

48

49

50

51

52

53

54

55

56

57

58

59

60
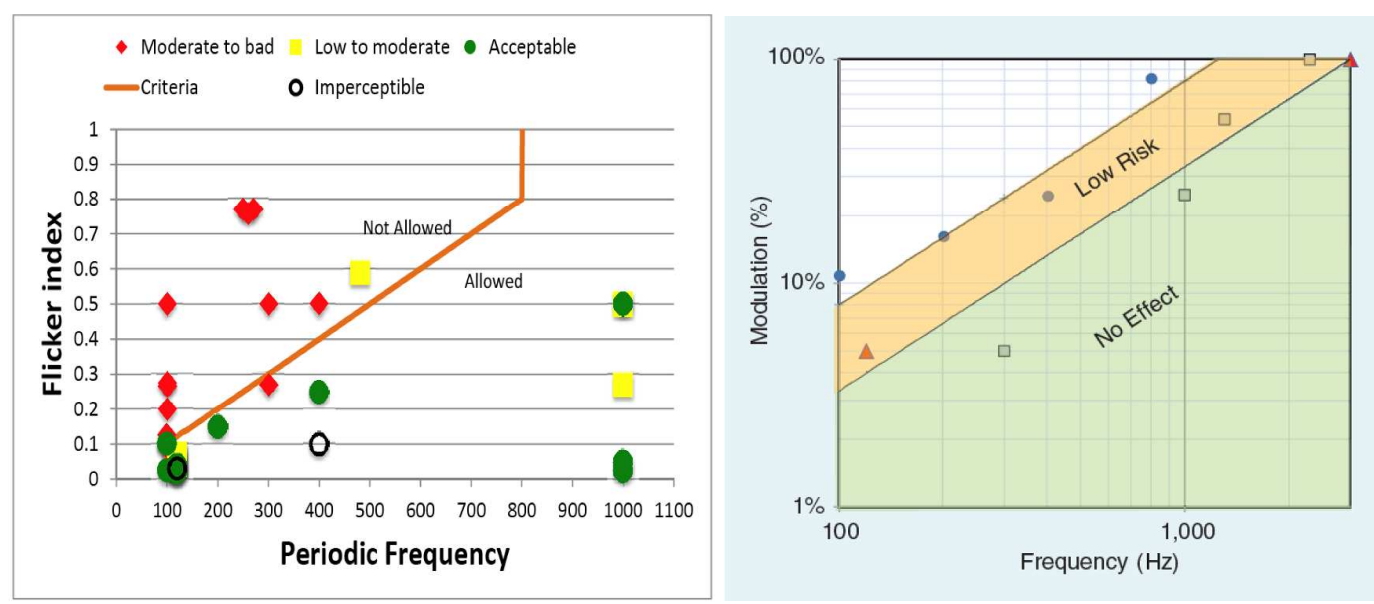

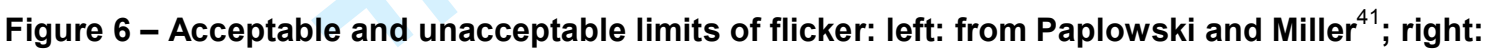
from Lehman and Wilkins ${ }^{42}$. Note the linear axes on the left figure and logarithmic axes on the right figure. Reproduced with permission. 


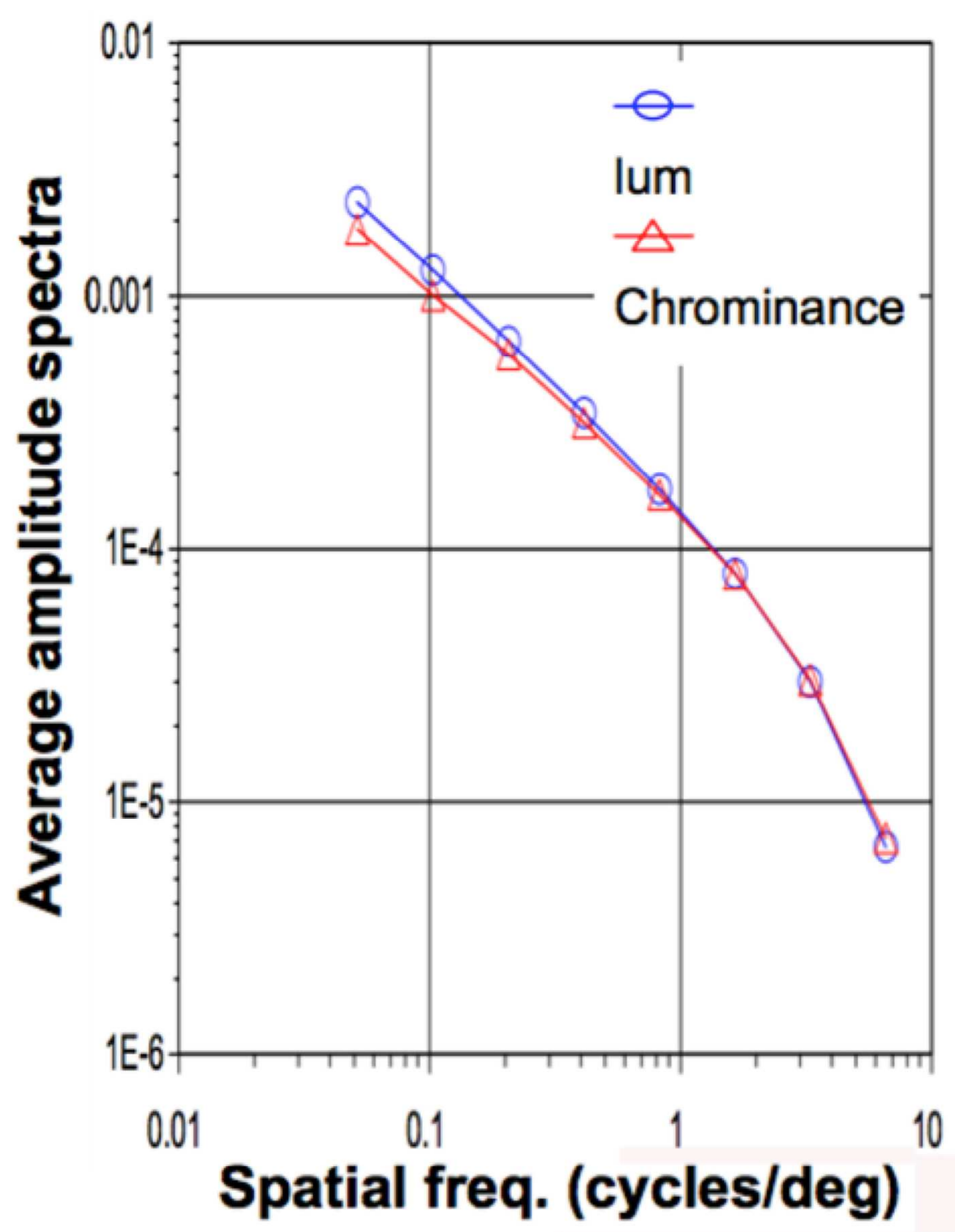

Figure $1-1 / f$ amplitude spectra for luminance and chrominance for the 29 scenes measured by Parraga et al.3

$169 \times 220 \mathrm{~mm}(144 \times 144$ DPI $)$ 


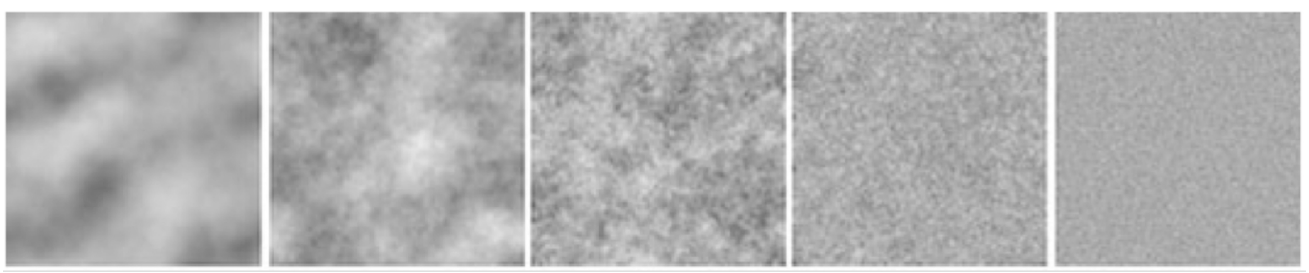

Figure 2 - Examples of meaningless patterns of filtered random dots. The slopes of the amplitude spectra (left-right) are $-2,-1.5,-1,-0.5$ and 0 (From Jurecevic et al. 12). $211 \times 42 \mathrm{~mm}(300 \times 300$ DPI $)$ 

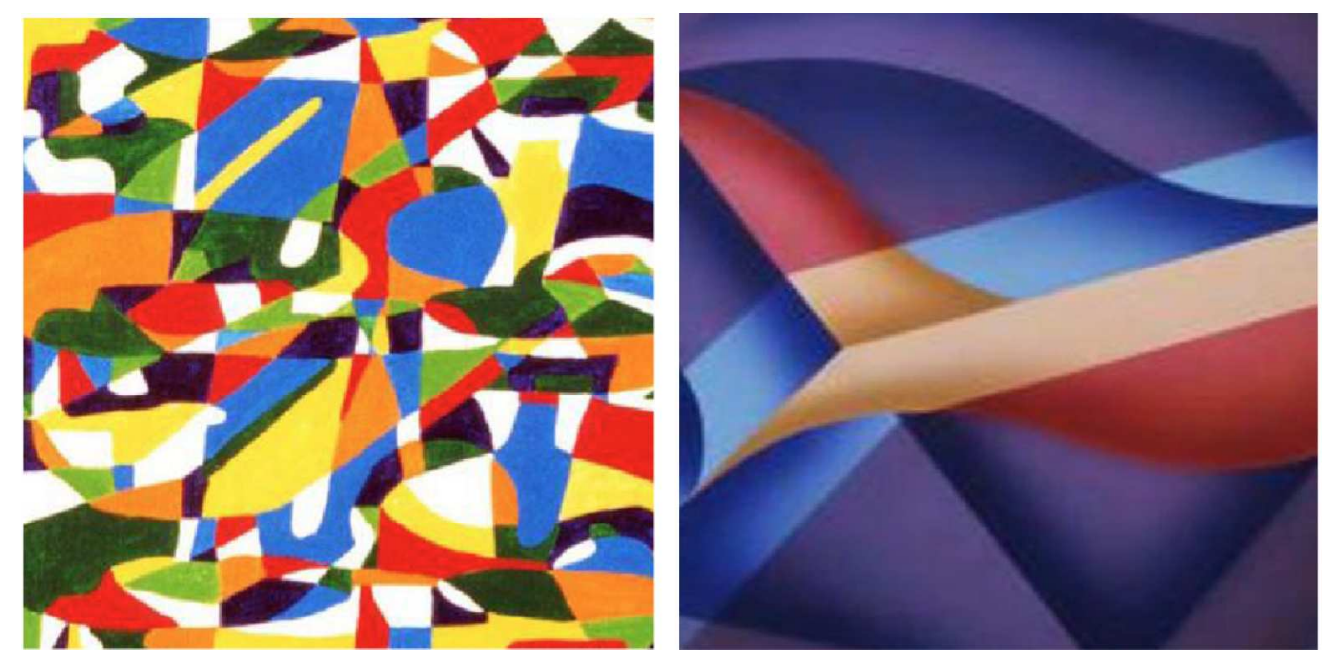

Figure 3 - Examples of artwork by Debbie Ayles that is recognized as uncomfortable (left) and comfortable (right) by the algorithm of Penacchio and Wilkins.17 $178 \times 87 \mathrm{~mm}(300 \times 300 \mathrm{DPI})$ 
Figure 4 - (left) Strong colour contrasts in a primary school (Courtesy of EME Furniture, reproduced with permission); (right) same figure with contrasts reduced. $208 \times 82 \mathrm{~mm}(300 \times 300$ DPI $)$ 

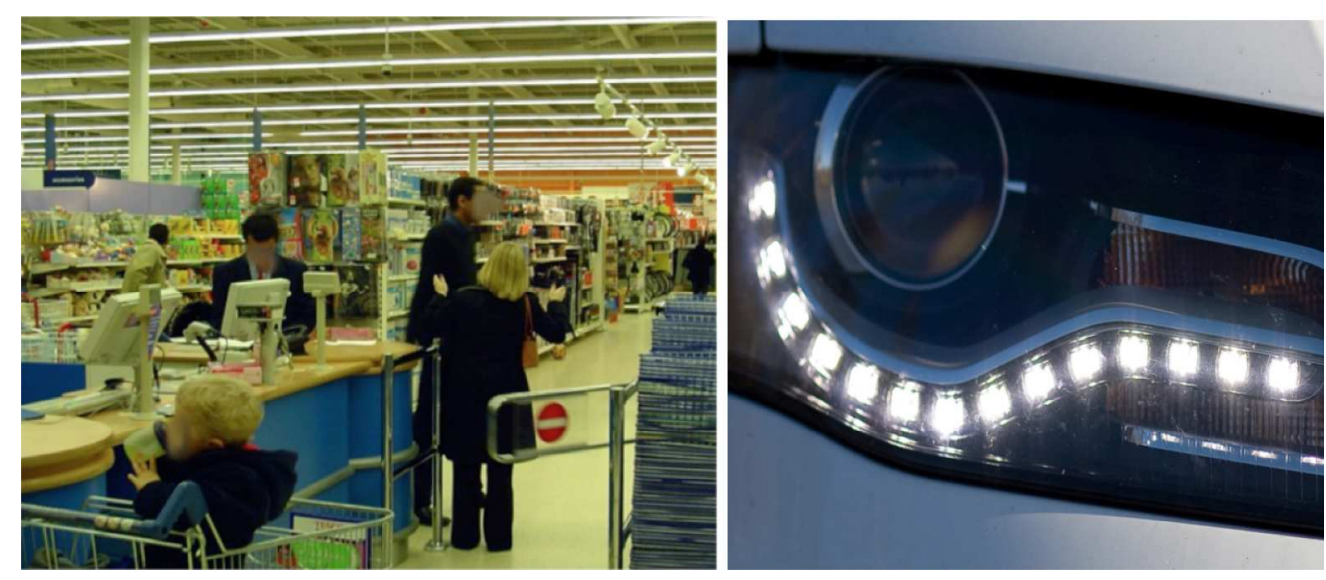

Figure 5 - Uncomfortable configurations of luminaires and lamps. $211 \times 89 \mathrm{~mm}(300 \times 300 \mathrm{DPI})$ 
1

2

3

4

5

6

7

8

9

10

11

12

13

14

15

16

17

18

19

20

21

22

23

24

25

26

27

28

29

30

31

32

33

34

35

36

37

38

39

40

41

42

43

44

45

46

47

48

49

50

51

52

53

54

55

56

57

58

59

60
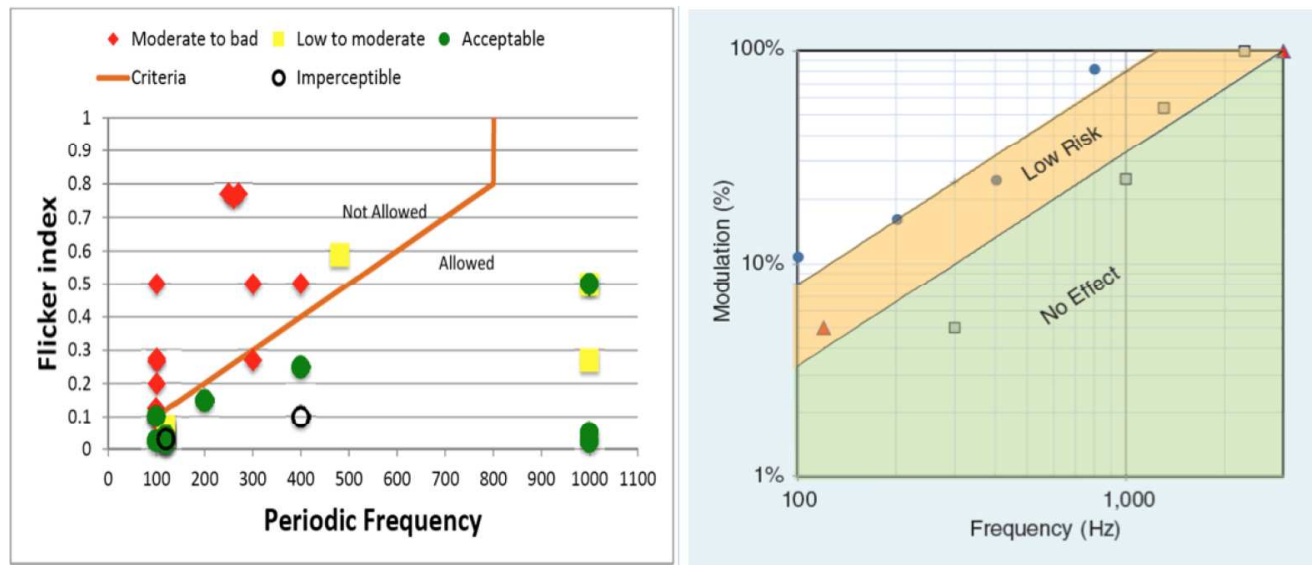

Figure 6 - Acceptable and unacceptable limits of flicker: left: from Paplowski and Miller 41; right: from Lehman and Wilkins 42. Note the linear axes on the left figure and logarithmic axes on the right figure. Reproduced with permission. $196 \times 83 \mathrm{~mm}(300 \times 300 \mathrm{DPI})$ 